\title{
Mineração
}

\section{Utilização de uma nova equação constitutiva para os evaporitos de Taquari- Vassouras}

\author{
Cláudio Lúcio Lopes Pinto \\ Engenheiro de Minas, PhD., Prof. Adjunto \\ Departamento de Engenharia de Minas - EEUFMG \\ E-mail: cpinto@demin.ufmg.br \\ Eliane Ribeiro da Cruz \\ Engenheira de Minas, MSc.
}

\section{Resumo}

As equações contitutivas Norton Power Law, que utilizam um e dois componentes (Norton, 1929), e a equação proposta por Pinto (1995), para representar o comportamento mecânico dos evaporitos da Mina de Boulby (Inglaterra), foram utilizadas para simular o fechamento da câmara experimental na Mina de Taquarí-Vassouras.

Os resultados obtidos para a lei Norton Power demonstraram o caráter linear da deformação representada por essa equação. Conseqüentemente, essas formulações não são capazes de representar a fluência primária (transiente) ou o efeito de endurecimento no tempo. A equação Bayle-Norton, utilizada por Quinteiro (1992), supera esse problema. Entretanto o termo tempo $(t)$, explícito em sua formulação, não permite a sua utilização em situações práticas encontradas em operações de mineração.

A equação proposta por Pinto (1995) apresentou resultados bastante satisfatórios na simulação realizada. Uma análise dessa equação mostra ser ela capaz de representar, tanto a fluência primária (transiente), quanto a secundária (steady-state).

Palavras-chave: fluência, lavra de evaporitos, equações constitutivas

\section{Abstract}

The constitutive equation Norton Power Law, using one and two components (Norton, 1929) and an equation proposed by Pinto (1995) to represent the mechanical behavior of Boulby Potash Mine evaporites were used to simulate the closure of an experimental panel excavated at Taquari-Vassouras Mine.

The results obtained with the Norton Power law showed the linear characteristic of the deformation computed by this equation. Therefore, such equations are not able to represent the transient (primary) creep or the time hardening effect. The Bayle-Norton constitutive law, used by Quinteiro (1992), overcomes this problem. Unfortunately, the time term ( $t)$, used explicit in its formulation, does not allow it to be used in practical mining situations.

The equation proposed by Pinto (1995) led to results considered very satisfactory in the simulation carried out. An analysis of this equation demonstrates its capability of simulating, both, the transient (primary) and the steady-state (secondary) creep.

Keywords: Creeping, evaporites mining, constitutive laws 


\section{Introdução}

O comportamento geomecânico particular dos evaporitos leva à pesquisa de novas equações, que permitam descrever o comportamento de fluência com maior representatividade e, assim, poder planejar a sua lavra com segurança e eficiência.

Várias equações constitutivas têm sido utilizadas para representar o comportamento mecânico de fluência dos evaporitos. Entre as mais utilizadas, estão as equações Norton Power Law para um componente e para dois componentes (Norton, 1929) e a equação de BayleNorton (Quinteiro, 1992). Todas essas equações, constitutivas possuem limitações que não permitem que fenômenos do comportamento de fluência observados in situ sejam reproduzidos corretamente. A equação proposta por Pinto (1995), utilizada com sucesso na mina de Boulby, na Inglaterra, tem como característica principal a capacidade de representar, em sua formulação, os fenômenos de endurecimento no tempo (strain hardening) e amolecimento no tempo (strain softening).

\section{O comportamento de fluência}

O comportamento de fluência é caracterizado pela deformação do maciço rochoso ao longo do tempo, mesmo quando submetido a valores constantes de tensão relativamente baixos. A curva de fluência é dividida em três regiões, como pode ser visto na Figura 1. A região I é a região da fluência primária ou transiente, que se caracteriza por um aumento na taxa de deformação ao longo do tempo; a região II é a região de fluência secundária, que se caracteriza por uma taxa de deformação constante ao longo do tempo e a região III é a região da fluência terciária, que se caracteriza por um rápido aumento na taxa de deformação, ocasionando a ruptura do maciço. Os segmentos PQ e TU representam a recuperação instantânea da deformação elástica, quando a tensão aplicada for reduzida a zero. Já o segmento QR, como discutido por Jaeger e Cook (1971), mostra que, se a redução da tensão acon- tecer na região da fluência transiente, a recuperação da deformação tenderá a zero, ou seja, não haverá deformação permanente. Esse fenômeno é chamado de visco-elasticidade ou elasticidade dependente do tempo. Já o caminho UV mostra que, se a redução da tensão aplicada ocorrer na região II, a deformação final tenderá a um valor de deformação permanente (Jeremic, 1987).

Alguns pesquisadores discordam dessa afirmação. Eles relatam que, no caminho TUV, a taxa de fluência diminuiria constantemente e a curva aproximaria de zero, caracterizando o comportamento de endurecimento no tempo (time hardening) (Baar, 1977).

\section{Equações constitutivas do comportamento de fluência}

\subsection{Equação constitutiva Norton Power Law}

A lei constitutiva Norton Power
Law é uma equação muito simples e, por isto, muito utilizada. Essa formulação possui apenas duas constantes a serem determinadas, como pode ser visto na equação 1 (Norton, 1929).

$\dot{\varepsilon}=\mathrm{a} \bar{\sigma}^{\mathrm{n}}$

Onde:

$\dot{\varepsilon}$ - taxa de deformação por fluência.

$\bar{\sigma}$ - tensão de Von Mise.

a e $\mathrm{n}$ - constantes relativas ao material.

Apesar dessa simplicidade, devese ter cuidado ao se definirem os valores das constantes $a$ e $n$. Diversos trabalhos encontrados na literatura não levam em consideração as equações de equilíbrio e definem tais valores sem considerar essa condição.

Sempre que uma escavação é introduzida em um meio, as tensões primitivas são perturbadas e uma nova distribuição de tensão é alcançada. As tensões, nesse novo estado, são denominadas tensões totais. A diferença entre as tensões totais e as tensões primitivas

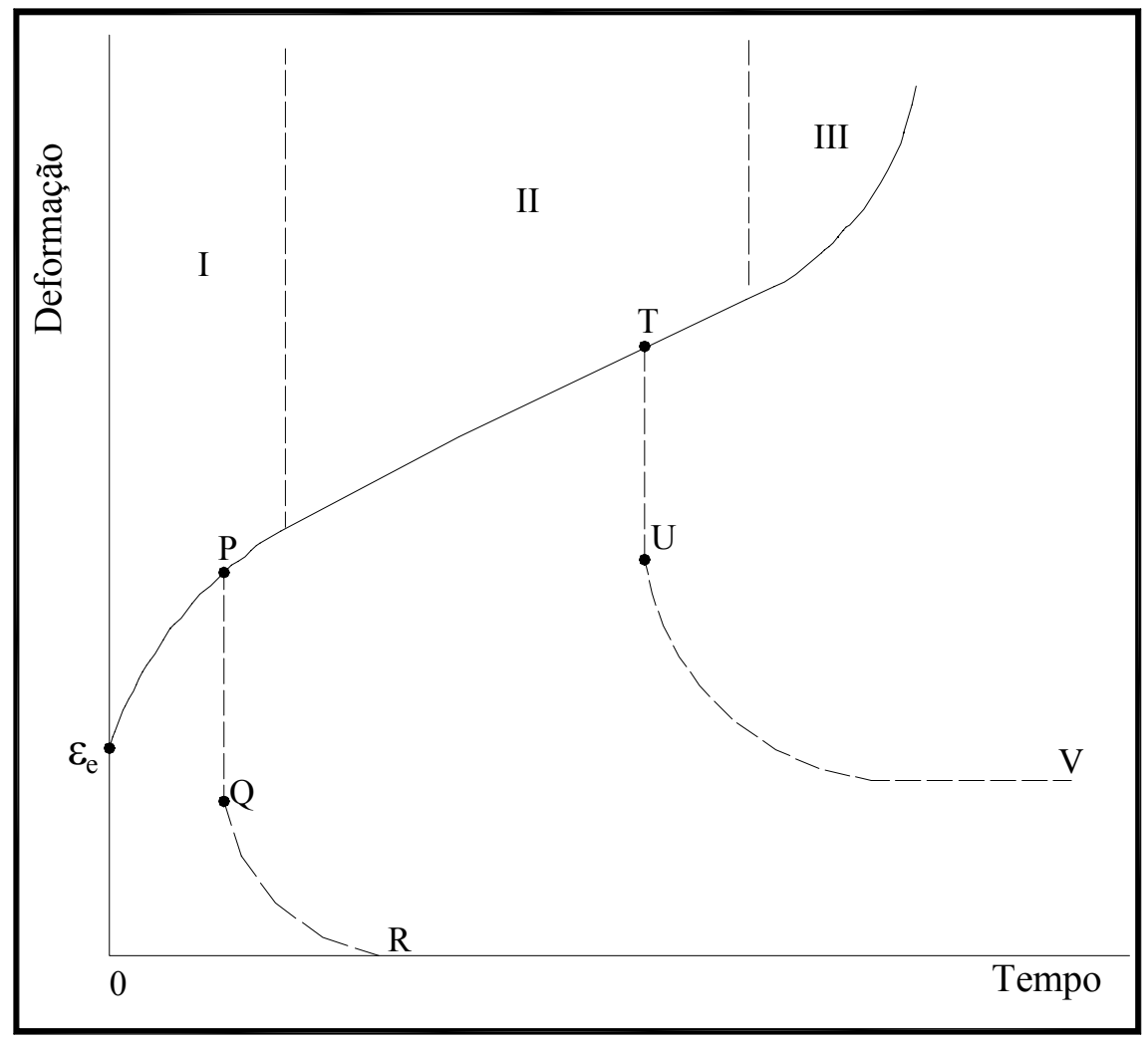

Figura 1 - Curva típica de fluência (Jaeger e Cook, 1971). 
correspondentes denomina-se tensões induzidas. Para se obter o equilíbrio das forças, é necessário assegurar que a resultante das tensões induzidas em todo o domínio seja nula (Cruz, 2003).

Em seu estudo, Pinto (1995) chegou à conclusão que a equação de Norton atende às condições de equilíbrio apenas quando o valor atribuído à constante $\mathrm{n}$ estiver situado no intervalo $1 \leq \mathrm{n} \leq 2$.

Uma outra limitação dessa equação é o fato de ser linear. Sendo assim, ela representa apenas a fluência secundária, que se caracteriza por uma taxa de deformação constante ao longo do tempo.

A equação constitutiva Norton Power Law para dois componentes é utilizada para representar, tanto a fluência primária, quanto a secundária, definindo-se uma tensão de referência (Itasca, 1996). Entretanto uma observação mais detalhada mostra que, segundo a equação de Norton, a fluência ocorre sob tensão constante, o que invalida a utilização de uma tensão de referência. Além disso, utilizando essa abordagem, a curva de fluência seria representada por duas retas com inclinações diferentes (Cruz e Pinto, 2002).

\subsection{Equação de Bayle-Norton}

A equação de Bayle-Norton foi proposta com o objetivo de representar o fenômeno de endurecimento no tempo. Entretanto sua aplicação tem limitações impostas pela utilização explícita do tempo em sua formulação:

$\dot{\varepsilon}=\mathrm{a} \bar{\sigma}^{\mathrm{n}} \mathrm{t}^{\mathrm{m}}$

Onde: $t$ é o tempo e $m$ é uma constante relativa ao material (Quinteiro, 1992).

A utilização do tempo, nessa equação, limita a simulação de escavações contíguas, realizadas em tempos diferentes. Não há informações de como considerar a variável tempo na introdução da nova escavação.

\subsection{Equação proposta}

A nova equação proposta para os evaporitos de Taquari-Vassouras foi proposta por Pinto (1995), para representar o comportamento mecânico dos evaporitos da mina de Boulby. Essa equação (3), não linear, é capaz de representar, tanto a fluência primária, quanto a secundária por meio de uma única curva.

$$
\dot{\varepsilon}=\frac{\mathrm{a} \bar{\sigma}^{\mathrm{n}}}{(\gamma+\bar{\varepsilon})^{\beta}}
$$

Nessa equação, $\gamma$ e $\beta$ representam variáveis do material e $\bar{\varepsilon}$ representa a deformação de Von Mise. A constante $\gamma$ assegura uma transição suave entre a lei Norton Power $(\gamma>>\bar{\varepsilon})$ e a lei de endurecimento no tempo $(\gamma<<\bar{\varepsilon})$.

\section{Aplicação das equações}

Com o objetivo de estudar as equações constitutivas Norton Power para um e dois componentes e a nova equação proposta, foi criado um modelo numérico representativo do ciclo deposicional VII da região da sub-bacia de TaquariVassouras, onde estão localizados os evaporitos da região, inclusive a silvini- ta, que é lavrada pela CVRD. O modelo representa uma câmara e um painel experimentais separados por um pilar de $57 \mathrm{~m}$ de largura. As medidas reais de convergência da câmara experimental foram comparadas com as reproduzidas pelo modelo para efeito de calibração e verificação da validade das equações (Cruz, 2001).

Todos os outros parâmetros de comportamento mecânico, diferentes daqueles empregados nas equações constitutivas em estudo, foram os mesmos utilizados por outros autores.

\section{Resultados}

Inicialmente foi testada a equação constitutiva Norton Power Law para um componente, observando-se as restrições, para a constante $n$, definidas pela condição de equilíbrio, ou seja, $1 \leq \mathrm{n} \leq 2$.

Os melhores resultados são representados na Figura 2, obtido com os valores apresentados na Tabela 1. Esse gráfico confirma o que foi discutido anteriormente a respeito da linearidade da equação constitutiva Norton Power Law.

Em seguida foi utilizada a equação constitutiva Norton Power Law para dois componentes. A utilização dessa equa-

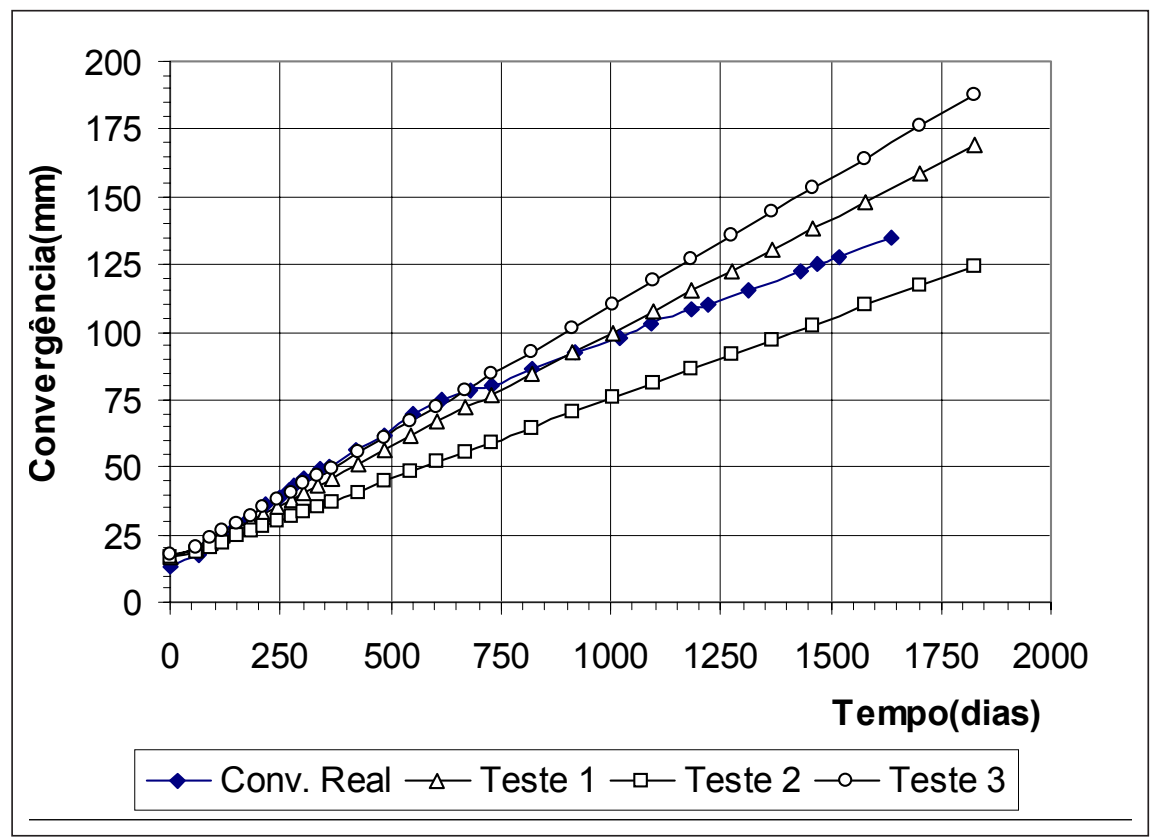

Figura 2 - Convergência da câmara experimental segundo a equação constitutiva Norton Power Law para um componente. 
Utilização de uma nova equação constitutiva para os evaporitos de Taquari-Vassouras

ção forneceu resultados parecidos com aqueles obtidos com a equação para um componente. Pode-se também observar que a mudança de inclinação da reta, referente à tensão de referência, não foi verificada durante a simulação (Figura 3 e Tabela 2).

A utilização da nova equação proposta forneceu resultados bastante satisfatórios (Figura 4 e Tabela 3). Com essa equação, foi possível simular o comportamento de fluência observado in situ, em toda sua totalidade, observando a redução gradativa da taxa de deformação (time ou strain hardening).

\section{Conclusões}

A utilização da equação constitutiva Norton Power Law, para um componente, não foi capaz de representar corretamente o fenômeno de endurecimento no tempo observado na câmara experimental da Mina de Taquari-Vassouras. Isto se deve ao fato de essa equação representar uma razão de deformação constante no tempo. Assim, essa equação constitutiva não deve ser utilizada para representar o comportamento de fluência dos evaporitos da região de Taquari-Vassouras.

A equação constitutiva Norton Power Law, para dois componentes, utiliza duas retas para simular, aproximadamente, o fenômeno de endurecimento no tempo. O critério de transição entre as retas é um valor de tensão de referência. Porém, segundo a lei de Norton Power, o estado de tensão existente no maciço rochoso não varia durante a fluência. Dessa forma, apenas uma reta é representada, como na equação para um componente. Assim, essa equação também não pode ser utilizada para representar o comportamento mecâncico dos evaporitos da sub-bacia de Taquari-Vassouras.

A equação proposta por Pinto (1995) foi capaz de representar o comportamento mecânico dos evaporitos da sub-bacia de Taquari-Vassouras, simulando corretamente o fenômeno de endurecimento no tempo, observado na câmara experimental escavada na Mina (Cruz, 2003).
Tabela 1 - Valores de a e n para a equação Norton Power Law, para um componente.

\begin{tabular}{c|c|c|c|c|c|c}
\hline \multirow{2}{*}{ Litologia } & \multicolumn{2}{|c|}{ Teste 1 } & \multicolumn{2}{c|}{ Teste 2 } & \multicolumn{2}{c}{ Teste 3 } \\
\cline { 2 - 7 } & $\mathbf{a}$ & $\mathbf{n}$ & $\mathbf{a}$ & $\mathbf{n}$ & $\mathbf{a}$ & $\mathbf{n}$ \\
\hline Silvinita & $5,0 \times 10^{-13}$ & 1,4 & $5,0 \times 10^{-13}$ & 1,3 & $8,0 \times 10^{-13}$ & 1,3 \\
\hline Halita & $5,0 \times 10^{-13}$ & 1,4 & $5,0 \times 10^{-13}$ & 1,3 & $8,0 \times 10^{-13}$ & 1,3 \\
\hline Taquidrita & $1,0 \times 10^{-9}$ & 1,6 & $1,0 \times 10^{-9}$ & 1,5 & $1,0 \times 10^{-9}$ & 1,5 \\
\hline Carnalita & $8,0 \times 10^{-10}$ & 1,6 & $8,0 \times 10^{-10}$ & 1,5 & $8,0 \times 10^{-10}$ & 1,5 \\
\hline
\end{tabular}

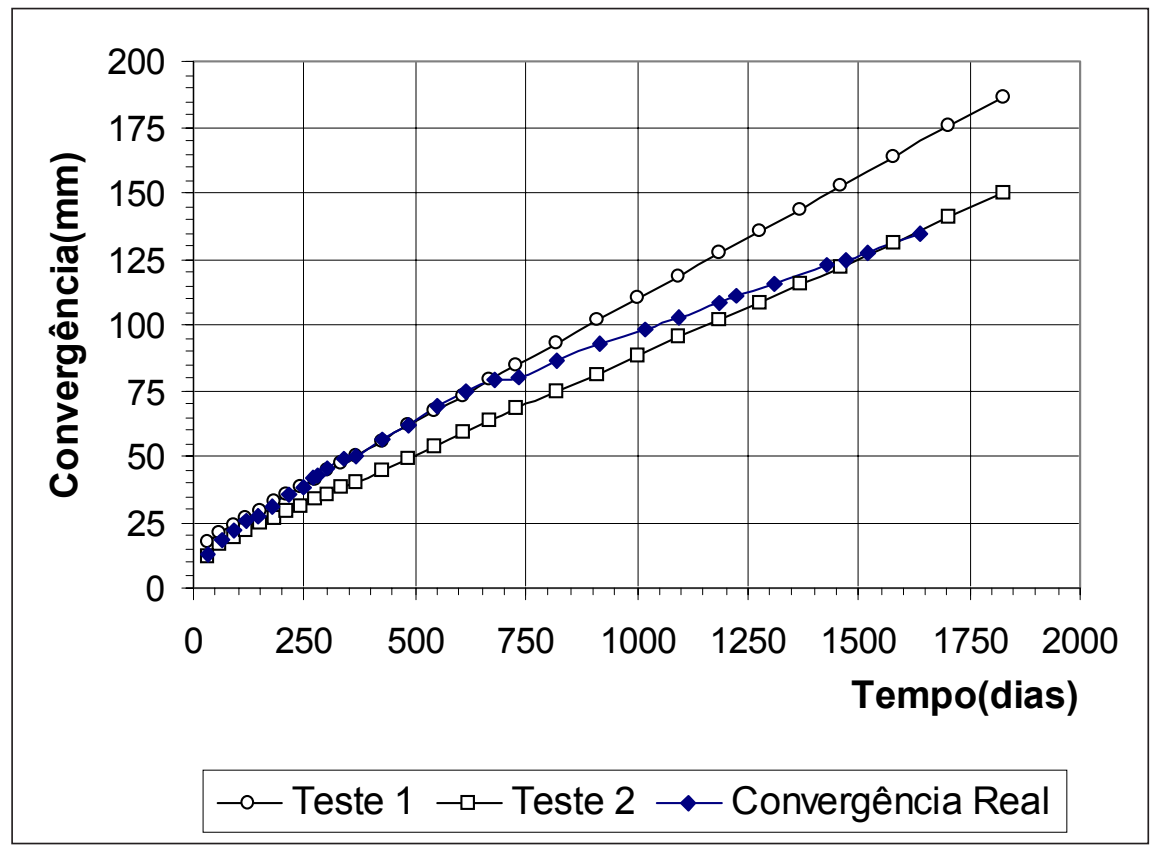

Figura 3 - Convergência da câmara experimental segundo a equação constitutiva Norton Power Law, para dois componentes.

Tabela 2 - Valores de a e n para a equação Norton Power Law, para dois componentes.

\begin{tabular}{c|c|c|c|c|c|c|c|c}
\hline \multirow{2}{*}{ Litologia } & \multicolumn{3}{|c|}{ Teste 1 $\left(\sigma_{\text {ref }}=\mathbf{2 5 , 0} \mathbf{M P a}\right)$} & \multicolumn{3}{c|}{ Teste 2 ( $\left.\sigma_{\text {ref }}=\mathbf{2 5 , 5} \mathbf{M P a}\right)$} \\
\cline { 2 - 9 } & $\mathbf{a}_{1}$ & $\mathbf{n}_{1}$ & $\mathbf{a}_{\mathbf{2}}$ & $\mathbf{n}_{\mathbf{2}}$ & $\mathbf{a}_{1}$ & $\mathbf{n}_{1}$ & $\mathbf{a}_{\mathbf{2}}$ & $\mathbf{n}_{\mathbf{2}}$ \\
\hline Silvinita & $6,0 \times 10^{-13}$ & 1,4 & $5,0 \times 10^{-13}$ & 1,2 & $6,0 \times 10^{-13}$ & 1,4 & $6,0 \times 10^{-14}$ & 1,5 \\
\hline Halita & $6,0 \times 10^{-13}$ & 1,4 & $5,0 \times 10^{-13}$ & 1,2 & $6,0 \times 10^{-13}$ & 1,4 & $6,0 \times 10^{-14}$ & 1,5 \\
\hline Taquidrita & $8,0 \times 10^{-9}$ & 1,5 & $7,0 \times 10^{-9}$ & 1,4 & $8,0 \times 10^{-9}$ & 1,5 & $4,0 \times 10^{-9}$ & 1,6 \\
\hline Carnalita & $5,0 \times 10_{-9}$ & 1,5 & $3,0 \times 10^{-9}$ & 1,4 & $5,0 \times 10^{-9}$ & 1,5 & $2,5 \times 10^{-9}$ & 1,6 \\
\hline
\end{tabular}

106 REM: R. Esc. Minas, Ouro Preto, 56(2): 103-107, abr. jun. 2003 


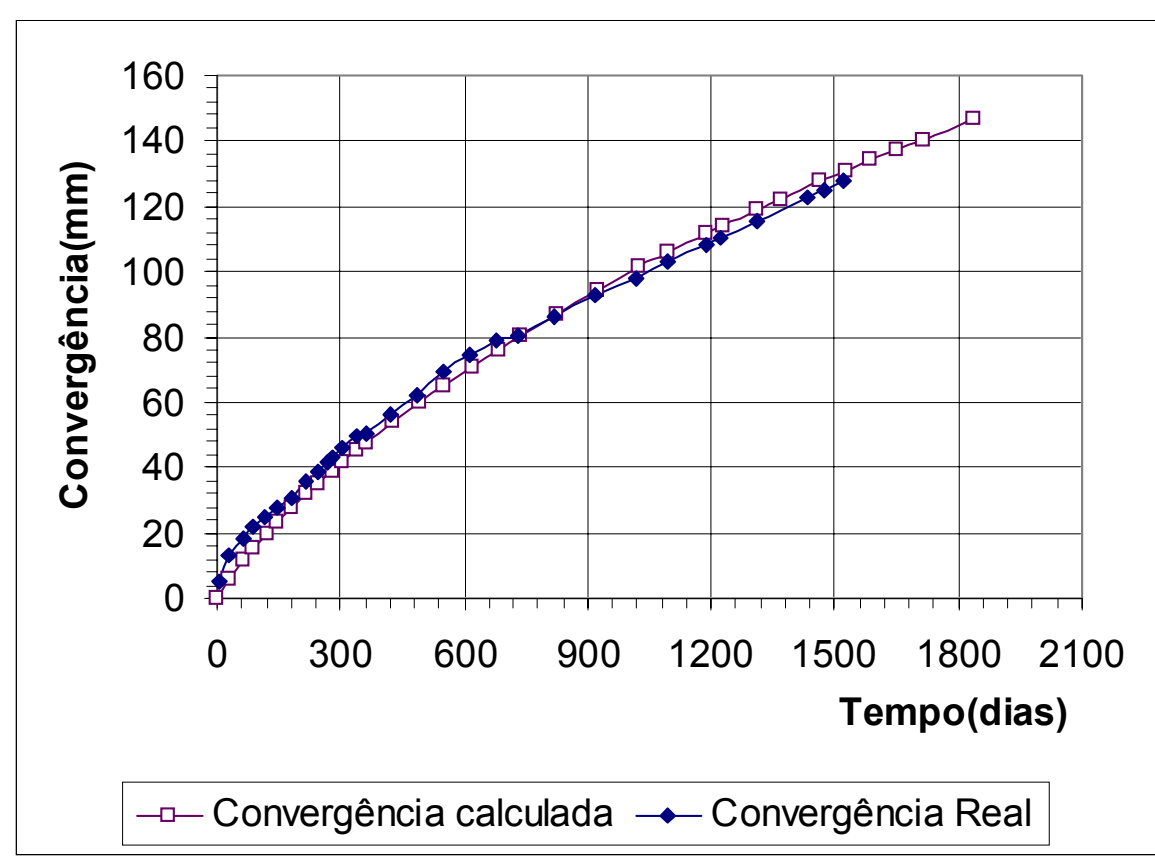

Figura 4 - Convergência da câmara experimental segundo a equação constitutiva proposta.

Tabela 4 - Valores das constantes da equação proposta.

\begin{tabular}{c|c|c|c|c}
\hline Litologia & $\mathbf{a}$ & $\mathbf{n}$ & $\gamma$ & $\beta$ \\
\hline Silvinita & $1,5 \times 10^{-21}$ & 1,80 & 0,12 & 9,75 \\
\hline Halita & $1,5 \times 10^{-21}$ & 1,80 & 0,12 & 9,75 \\
\hline Carnalita & $5,2 \times 10^{-15}$ & 1,85 & 0,12 & 3,00 \\
\hline Taquidrita & $6,5 \times 10^{-15}$ & 1,90 & 0,12 & 3,00 \\
\hline
\end{tabular}

\section{Referências \\ Bibliográficas}

BAAR, C. A., Applied Salt-Rock Mechanics, Part 1. Amsterdam: Elsevier Sci-entific Publishing Company, 1977.

CRUZ, E. R., PINTO, C. L. L. Modelagem numérica de escavações subterrâneas em evaporitos da sub-bacia de TaquariVassouras. In: CONGRESSO BRASILEIRO DE MINA A CÉU ABERTO E CONGRESSO BRASILEIRO DE MINA SUBTERRÂNEA, 2. Belo Horizonte. 2002.

CRUZ, E. R. Modelagem numérica de escavações subterrâneas em evaporitos da sub-bacia de Taquari-Vassouras. Belo Horizonte: Escola de Engenharia da UFMG, 2003. 98p. (Dissertação de Mestrado em Engenharia de Minas).

ITASCA. Manual for FLAC (Fast Lagrangian Analysis of Continua). Minneapolis: Minnesota Itasca Consulting Group, 1996.

JAEGER, J. C., COOK, N. G. W., Fundamentals of rock mechanics. Londres: Chapman and Hall Ltd. and Science Paper Backs, 1971.

JEREMIC, M. L. Ground mechanics in hard rock mining, 1987, p. 38-42.

NORTON F. H. Creep of steel at high temperatures. New York: MacGraw-Hill, 1929.

PINTO, C. L. L. Longwall mining in Boulby Potash Mine: a numerical study. USA: Colorado School of Mines, 1995. (Tese de Doutorado).

QUINTEIRO, C. R. Numerical modeling of time dependent ground behavior around a deep potash mine in preparation for layout design. USA: Colorado School of Mines, 1992. (Tese de Doutorado).

Artigo recebido em 05/09/2003 e aprovado em 19/05/2004.

\section{REM: a mais antiga revista técnico- científica do setor mínero-metalúrgico.}

$* * * * * *$

\section{Assine e Publique}

$\star * * * * *$ 